Supporting Information

\title{
Engineering peptide-functionalized biomimetic nanointerfaces for synergetic capture of circulating tumor cells in an EpCAM-independent manner
}

Huifei Zhong, ${ }^{\dagger a, c}$ Chunwang Yuan, ${ }^{\dagger b}$ Jiayuan He, ${ }^{a, c}$ Yang Yu, ${ }^{a, c}$ Yulong Jin, ${ }^{a, c}$ Yanyan Huang ${ }^{* a, c}$ and Rui Zhao ${ }^{a, c}$

a. Beijing National Laboratory for Molecular Sciences, CAS Key Laboratory of Analytical Chemistry for Living Biosystems, CAS Research/Education Center for Excellence in Molecular Sciences, Institute of Chemistry, Chinese Academy of Sciences, Beijing 100190, China

b. Center of Interventional Oncology and Liver Diseases, Beijing Youan Hospital, Capital Medical University, Beijing 100069, China

c. University of Chinese Academy of Sciences, Beijing 100049, China

$\dagger$ These authors contributed equally to this work.

*E-mail: yyhuang@iccas.ac.cn 


\section{Table of Content}

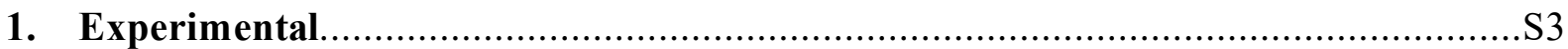

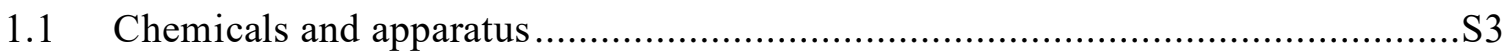

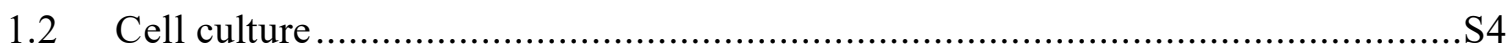

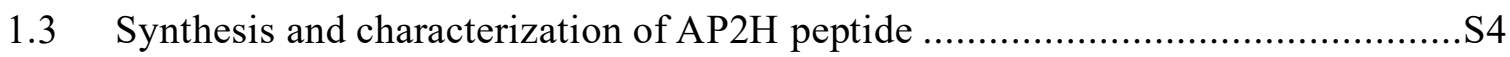

1.4 Determination of binding constants toward cancer cells ...................................S5

1.5 Analysis of EpCAM expression with western blot and immunofluorescence staining

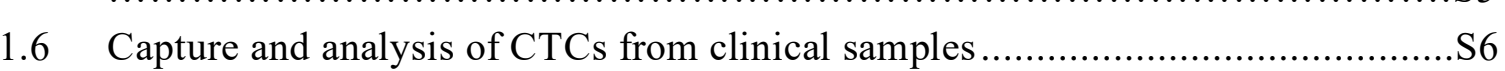

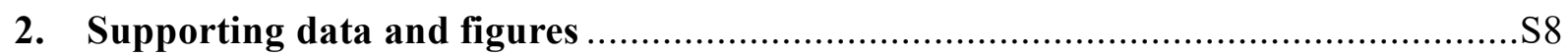

2.1 Synthesis and characterization of peptide-functionalized nanointerfaces .............S8

2.2 Fabrication of control substrates and their cell capture performance ..................S11

2.3 Cell binding kinetics on Si@PLGA-NF@AP2H substrate.................................. 12

2.4 Characterization of LAPTM4B and EpCAM in HEK293 and HCC cell lines ....S13

2.5 Determination of peptide binding affinity toward HuH-7 and Hep3B cells ........S 14

2.6 Characterization of EpCAM in MCF-7 and U251 cells ..................................S15

2.7 Capture of HCC cells with different EpCAM levels from whole blood ..............S15

2.8 Ligand exchange and cell release efficiencies of GSH..................................... 16

2.9 In vitro culture and proliferation activity of released cells ............................S18

2.10 CTC and CTC clusters captured from the blood samples of HCC patients .........S18

2.11 Analysis of CTCs captured from patient blood samples based on EpCAM level S19

2.12 LAPTM4B expression in CTCs captured from patient blood samples............... S20

2.13 Release of CTCs captured from patient blood samples ..................................S21

2.14 Characterization of LAPTM4B expression in CTCs captured from patients during therapy S21 


\section{Experimental}

\subsection{Chemicals and apparatus}

Chemicals. 9-Fluorenylmethoxycarbonyl (FMOC)-protected amino acids and 1Hydroxybenzotriazole (HOBt) were from Siam (Chicago, USA). FMOC-Gly-Wang Resin and 2-(1H-benzotriazole-1-yl)-1,1,3,3-tetramethyluronium hexafluorophosphate (HBTU) were from GL Biochem (Shanghai, China). $N, N$-dimethylformamide (DMF), acetonitrile and triisopropylsilane (TIS) were from Thermo Fisher Scientific (Waltham, USA). Trifluoroacetic (TFA), $N$-methylmorpholine, $N$-(3-dimethylaminopropyl)- $N$ '-ethylcarbodiimide hydrochloride (EDC), glutaraldehyde, glutathione (GSH), $N, N^{\prime}$-diisopropylethylamine (DIPEA), tween 20 and fluorescein isothiocyanate isomer I (FITC) were from J\&K Chemicals (Beijing, China). Piperidine, alcohol and ether were of analytical grade and from Beijing Chemical Factory (China). Dichloromethane, methanol and dimethylsulfoxide (DMSO) were from Concord Technology Co., Ltd. (Tianjin, China). Poly(D,L-lactide-co-glycolide) (PLGA, M. W. 3800054000), 1,1,1,3,3,3-hexafluoro-2-propanol and $N$-succinimidyl 3-(2-pyridyldithio)propionate (SPDP) were from Aladdin (Shanghai, China). $N$-hydroxysuccinimide (NHS), cysteamine and single cell whole genome amplification (SCWGA) kit were from Sigma-Aldrich (St. Louis, USA). Paraformaldehyde was from Yili Fine Chemicals Co., Ltd. (Beijing, China). Dulbecco's Modified Eagle's Medium (DMEM), Roswell Park Memorial Institute (RPMI) 1640 and phosphate buffered saline (PBS) were from Invitrogen (CA, USA). Fetal bovine serum (FBS) and $0.25 \%$ trypsin-EDTA was from Thermo Fisher Scientific (Waltham, USA). 4'6-diamidino2-phenylindole (DAPI) and 2'-(4-ethoxyphenyl)-5-(4-methyl-1-piperazinyl)-2,5'-bi-1Hbenzimidazole (Hoechst 33342) were from Solarbio (Beijing, China). Acridine orange (AO), propidium iodide (PI), and Calcein-AM were from Invitrogen (CA, USA). Bovine serum albumin (BSA) was from Biotech Pharmaceutical Co., Ltd. (Beijing, China). TritonX-100, RIPA lysis buffer, phenylmethanesulfonyl fluoride (PMSF), BCA protein assay kit, precast PAGE gel and Alexa Fluor 647-labeled goat anti-rabbit IgG were from Beyotime (Shanghai, China). Protease inhibitor was from BestBio (Shanghai, China). Polyvinylidene fluoride (PVDF) membrane was from Millipore (Bedford, USA). Antibody against $\beta$-actin was from Santa Cruz (Dallas, USA). Horseradish peroxidase (HRP)-labeled goat anti-rabbit or mouse IgG was from TransGen (Beijing, China). Chemiluminescence detection reagent was from EasyBio (Beijing, China). Antibody against LAPTM4B, antibody against EpCAM, FITC-labeled monoclonal antibody against CD45, eFluor ${ }^{\mathrm{TM}}$ 570-labeled monoclonal antibody against Cytokeratin (CK) and Alexa Fluor 488-labeled goat anti-rabbit IgG were from Abcam (Cambridge, UK). Ultra- 
pure water from a Milli-Q water purification system (Millipore, Bedford, USA) was used throughout.

Apparatus. A Shimadzu Prominence HPLC system (Kyoto, Japan) was used for the analysis of the synthesized peptide AP2H and FITC-labeled AP2H. Peptide purification was performed on a Shimadzu LC-20AR preparative HPLC system (Kyoto, Japan). High-resolution mass spectra was recorded on a Solarix 9.4T AS FTICR MS equipped with a MALDI source (Bruker Daltonics, Bremen, Germany). A BD Accuri C6 flow cytometry (USA) was used for the determination of binding affinities of AP2H peptide toward target cells. Binding affinities of Si@PLGA-NF@AP2H toward cells were measured on a SpectraMAX M2e microplate reader (Molecular Devices, USA). Fluorescence imaging was performed on an Olympus FV 1000IX81 confocal laser scanning microscopy (CLSM, Tokyo, Japan). Scanning Electron Microscopy (SEM) was carried out on a JSM-6700F scanning electron microscope (JEOL, Tokyo, Japan). Environment scanning electron microscopy (ESEM) was taken by Quanta FEG 250 emission environment scanning electron microscope (FEI, USA). Contact angle was measured on OCA25 contact angle system (DATAPhysics, German).

\subsection{Cell culture}

Human hepatic cancer cells (HepG2), human breast cancer cells (MCF-7), and human embryonic kidney cells (HEK293) were from Shanghai Institutes for Biological Sciences, Chinese Academy of Sciences (Shanghai, China). Human glioma cells (U251), Human hepatic cancer cells (HuH-7 and Hep3B) were from KeyGen BioTECH (Nanjing, China). HepG2, MCF-7, HEK293, U251 and HuH-7 cells were cultured in an incubator DMEM at $37{ }^{\circ} \mathrm{C}$ with $5 \% \mathrm{CO}_{2}$. The culture medium was prepared from DMEM added with 10\% FBS. Hep3B cells were cultured in an incubator RPMI 1640 at $37{ }^{\circ} \mathrm{C}$ with $5 \% \mathrm{CO}_{2}$. The culture medium was prepared from RPMI 1640 added with 10\% FBS.

\subsection{Synthesis and characterization of AP2H peptide}

AP2H peptide was synthesized using solid phase peptide synthesis (SPPS) approach (Scheme S1). 9-fluorenylmethoxycarbonyl (FMOC)-Gly-Wang resin was used as the starting material. Piperidine was used as the de-protection reagent. HBTU was used as the coupling reagent. Peptide chains were cleaved from the resins by using a cocktail containing $95 \%$ TFA, $2.5 \% \mathrm{H}_{2} \mathrm{O}$ and $2.5 \%$ TIS. The synthesized peptide was purified on a Shimadzu LC-20AR preparative HPLC system (Kyoto, Japan). Water with $0.1 \%$ TFA and acetonitrile with $0.1 \%$ TFA were used as mobile phases. Purification was performed on a Diamonsil C18 column $(250 \times 10.0 \mathrm{~mm}$ i.d.) at a flow rate of $3.0 \mathrm{~mL} \mathrm{~min}^{-1}$. Gradient: 0-25-25.01-30 $\mathrm{min}, 17 \%-35 \%-80 \%-80 \%$ acetonitrile 
containing $0.1 \%$ TFA. After purification, the purity of the peptide was verified on a shim-pack XR-ODS column $(75 \times 4.6 \mathrm{~mm}$ i.d. $)$ at a flow rate of $1.0 \mathrm{~mL} \mathrm{~min}^{-1}$. Gradient: $0-10-15 \mathrm{~min}, 5 \%$ 90\%-90\% acetonitrile containing $0.1 \%$ TFA. The purified peptide was characterized with high resolution mass spectrometer.

\subsection{Determination of binding constants toward cancer cells}

The binding constants of AP2H peptide toward HepG2, HuH-7 and Hep3B cells were measured with flow cytometry analysis. AP2H peptide was labeled with FITC to indicate the binding signal. AP2H peptide $(5.35 \mathrm{mg})$ and FITC $(2.49 \mathrm{mg})$ were dissolved in $200 \mu \mathrm{L}$ of DMF. Subsequently, $2 \mu \mathrm{L}$ of DIPEA was added into the above mixture. After being stirred at room temperature for $2 \mathrm{~h}, 800 \mu \mathrm{L}$ of PBS was added to the reaction mixture. The reaction mixture was purified on a Shimadzu LC-20AR preparative HPLC system (Kyoto, Japan). The purified peptide was verified with high resolution mass spectrometer.

To evaluate the binding performance of free AP2H peptide, cancer cells were firstly treated with $1 \%$ BSA for 60 min to block the non-specific binding sites. Then, cells were incubated with FITC-labeled AP2H at different concentrations on ice for $60 \mathrm{~min}$. After washing, the stained cells were re-suspended with PBS and subjected to flow cytometry analysis (BD Accuri C6, USA). Flow cytometric data were analyzed with the FlowJo software. The $K_{\mathrm{D}}$ values were obtained with the SigmaPlot software by fitting the normalized fluorescence intensity $(\mathrm{Y})$ on the concentration of FITC-labeled $\mathrm{AP} 2 \mathrm{H}(\mathrm{X})$ according to the equation $\mathrm{Y}=\mathrm{B}_{\max } \mathrm{X} /\left(K_{\mathrm{D}}+\mathrm{X}\right)$.

For the determination of binding affinities of Si@PLGA-NF@AP2H toward target cells, HepG2 cells were pre-stained with Calcein-AM. Si@PLGA-NF@AP2H substrates with different concentrations of AP2H were respectively placed into the chamber of a 24-well plate. $1 \mathrm{~mL}$ pre-stained cell suspensions were added into the chamber for the incubation with the substrate. After $60 \mathrm{~min}$, the substrate was washed with PBS three times. The fluorescence intensity from the pre-stained HepG2 cells captured on Si@PLGA-NF@AP2H substrate was measured by a SpectraMAX M2e microplate reader (Molecular Devices, USA). The excitation wavelength was $490 \mathrm{~nm}$, and the emission wavelength was $515 \mathrm{~nm}$. The $K_{\mathrm{D}}$ values were obtained with the SigmaPlot software by fitting the normalized fluorescence intensity (Y) on the concentration of $\mathrm{AP} 2 \mathrm{H}(\mathrm{X})$ according to the equation $\mathrm{Y}=\mathrm{B}_{\max } \mathrm{X} /\left(K_{\mathrm{D}}+\mathrm{X}\right)$.

\subsection{Analysis of EpCAM expression with western blot and immunofluorescence staining}

Western blot analysis was employed for the characterization of EpCAM expression levels in different cells. Cells were lysed with RIPA lysis buffer supplemented with $1 \%$ protease 
inhibitor and $1 \% \mathrm{PMSF}$ on ice for $5 \mathrm{~min}$. After centrifugation at $4{ }^{\circ} \mathrm{C}$ for $10 \mathrm{~min}$, the cell lysates were collected for the determination of total protein concentration using BCA protein assay. Protein extracts were separated with SDS-PAGE and then transferred to the PVDF membrane. The membrane was blocked with $5 \%$ nonfat milk in TBST for $60 \mathrm{~min}$, and then incubated separately with anti-EpCAM antibody (1:1000) and anti- $\beta$-actin antibody (Santa Cruz, 1:1000) at $4{ }^{\circ} \mathrm{C}$ overnight. For HepG2, HuH-7 and Hep3B cells, the primary antibody was anti-EpCAM antibody (abcam, product code ab223582). For MCF-7 and U251 cells, a polyclonal antiEpCAM antibody (abcam, product code ab71916) was applied. Afterwards, the membrane was washed with TBST four times and incubated with horseradish peroxidase-labeled secondary antibodies (TransGen, 1:5000) at room temperature for $1 \mathrm{~h}$. After washed with TBST, the membrane was visualized using chemiluminescence detection reagent (EasyBio, China) and imaged on a Tanon 5200 Multi chemiluminescence system (Shanghai, China). EpCAM expression levels were analyzed using Image $\mathbf{J}$ software by using $\beta$-actin as the reference.

The expression of EpCAM on cultured cells and CTCs was analyzed using immunofluorescence staining. Cells were firstly fixed with $4 \%$ paraformaldehyde in PBS for $20 \mathrm{~min}$ and then washed with PBS. Then, cells were permeated with $0.1 \%$ TritonX-100 for 5 min and washed with PBS. After the blockage with 5\% BSA solution for $60 \mathrm{~min}$, cells were incubated with anti-EpCAM antibody at $4{ }^{\circ} \mathrm{C}$ overnight. For HepG2, HuH-7 and Hep3B cells, the primary antibody was anti-EpCAM antibody (abcam, product code ab223582). For MCF-7 and U251 cells, a polyclonal anti-EpCAM antibody (abcam, product code ab71916) was applied. Afterwards, cells were washed with PBST and incubated with Alexa Fluor 488-labeled goat anti-rabbit IgG at room temperature for $1 \mathrm{~h}$. Then, cells were washed with PBS and incubated with DAPI for $10 \mathrm{~min}$. The samples were imaged on CLSM with a UPLSAPO $100 \times$ oil-immersion objective (Olympus). Alexa Fluor 488 was excited by a FV5-LAMAR $488 \mathrm{~nm}$ laser and collected with a band-pass filter within the range of 500-600 nm. DAPI was excited by a $50 \mathrm{~mW}, 405 \mathrm{~nm}$ Laser Head FV 5-LD405-2 and collected with a band-pass filter within the range of $425-475 \mathrm{~nm}$.

\subsection{Capture and analysis of CTCs from clinical samples}

Whole blood samples of HCC patients (pathologically confirmed) were obtained from Beijing Youan Hospital, Capital Medical University. Blood samples from healthy donors and HCC patients were collected with informed consent and according to Institutional Guideline. Blood was collected into vacutainer tubes (BD Vacutainer EDTA blood collection tubes). Without pretreatment, the blood samples (average volume $0.7 \mathrm{~mL}$ ) were added into the chambers 
containing the substrate. After incubated for $60 \mathrm{~min}$, the blood was removed and the substrate was gently washed. The cells captured on the substrate were subjected to immunofluorescence staining to identify CTCs from blood cells. Briefly, cells were firstly fixed with $2.5 \%$ glutaraldehyde in PBS for $20 \mathrm{~min}$ and then washed with PBS. Next, the cells were permeated with TritonX-100 for 20 min followed by washed with PBS. Then, cells were blocked with 1\% BSA solution for $30 \mathrm{~min}$. Subsequently, cells were stained with FITC-labeled antibody against CD45, eFluor ${ }^{\mathrm{TM}}$ 570-labeled antibody against CK, and DAPI. Finally, cells were imaged on CLSM with a UPLSAPO $10 \times$ objective and a UPLSAPO $100 \times$ oil-immersion objective (Olympus). FITC was excited by a FV5-LAMAR $488 \mathrm{~nm}$ laser and collected with a band-pass filter within the range of 500-545 nm. eFluor ${ }^{\mathrm{TM}} 570$ was excited by a FV10-LD559 $559 \mathrm{~nm}$ laser and collected with a band-pass filter within the range of 570-670 nm. DAPI was excited by a $50 \mathrm{~mW}, 405 \mathrm{~nm}$ Laser Head FV5-LD405-2 and collected with a band-pass filter within the range of 425-475 $\mathrm{nm}$. The captured CTCs were also released from the substrate and collected into centrifuge tubes for downstream cellular and molecular analysis.

The expression of LAPTM4B on CTCs was analyzed using immunofluorescence staining. Cells were firstly fixed with $2.5 \%$ glutaraldehyde in PBS for $20 \mathrm{~min}$ and then washed with PBS. Next, cells were permeated with TritonX-100 for 20 min and then washed with PBS. Afterward, cells were blocked with 1\% BSA solution for $30 \mathrm{~min}$. Cells were incubated with anti-LAPTM4B antibody at $4{ }^{\circ} \mathrm{C}$ overnight. Then, cells were washed with PBS and incubated with Alexa Fluor 647-labeled goat anti-rabbit IgG at room temperature for $1 \mathrm{~h}$. After washed with PBS, cells were incubated with DAPI for $10 \mathrm{~min}$. The samples were imaged on CLSM with a UPLSAPO 100× oil-immersion objective (Olympus). Alexa Fluor 647 was excited by a FV10-LD635 635 $\mathrm{nm}$ laser and collected with a band-pass filter within the range of $650-750 \mathrm{~nm}$. DAPI was excited by a $50 \mathrm{~mW}, 405 \mathrm{~nm}$ Laser Head FV5-LD405-2 and collected with a band-pass filter within the range of $425-475 \mathrm{~nm}$.

For gene analysis of CTCs from clinical samples, the substrate was treated with GSH (1 mg $\mathrm{mL}^{-1}$, in PBS) for $30 \mathrm{~min}$ at room temperature to release the captured CTCs. The released CTCs were collected in a PCR tube and re-suspended in PBS. Afterwards, the whole genome of released CTCs was amplified according to the procedure of GenomePlex ${ }^{\circledR}$ Single Cell Whole Genome Amplification kit (Sigma-Aldrich, USA). TP53, CTNNB1 and KRAS mutations were analyzed using Sanger sequencing. 


\section{Supporting data and figures}

\subsection{Synthesis and characterization of peptide-functionalized nanointerfaces}

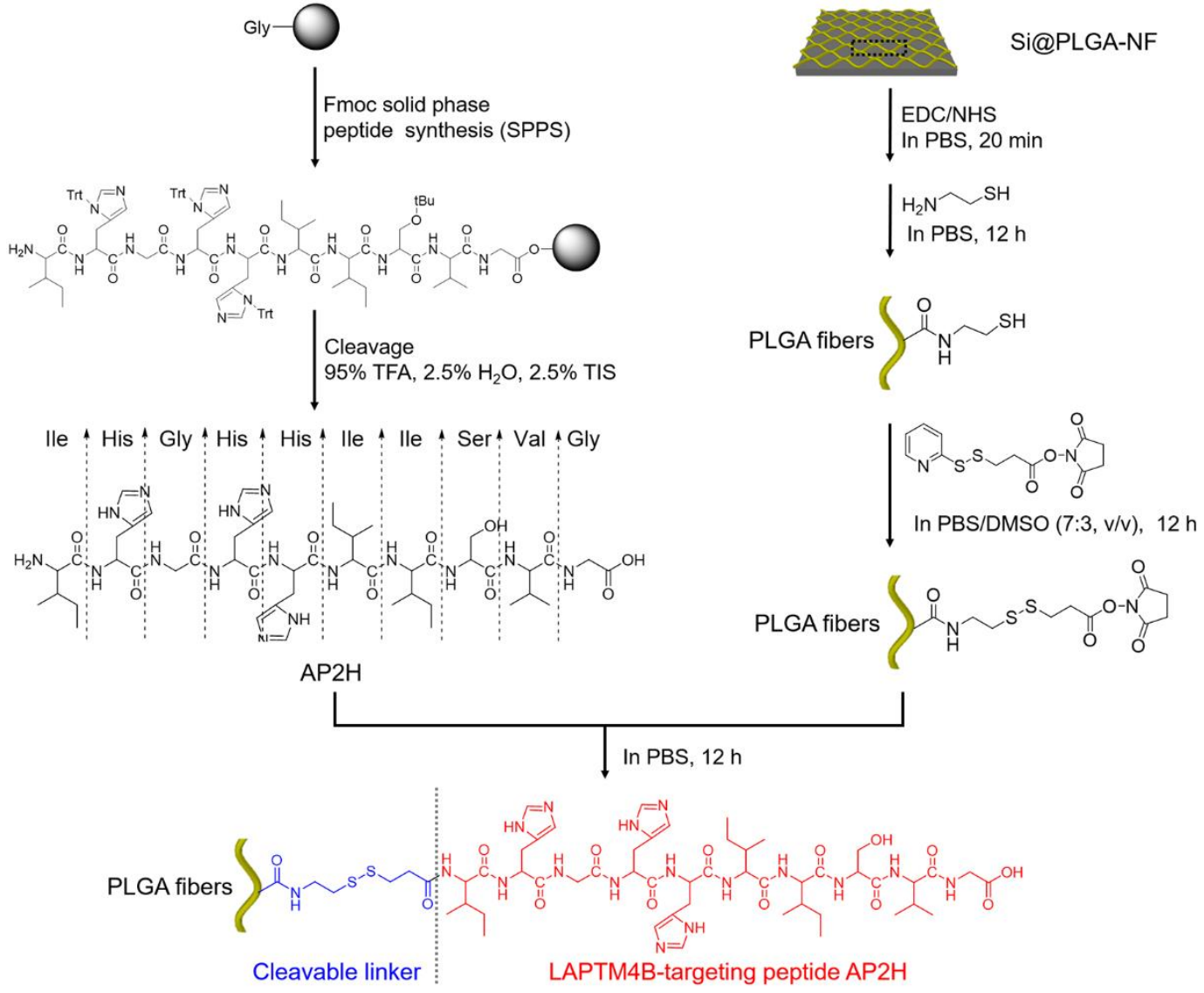

Scheme S1. Schematic illustration of the synthesis processes of AP2H and its immobilization onto the electrospun PLGA fibers.

The effects of PLGA concentration and flow rate on the diameters of nanofibers were examined. Different polymer concentrations $(3,7,10,13$ and $15 \%)$ and flow rates $\left(0.1,0.3\right.$ and $0.5 \mathrm{~mL} \mathrm{~h}^{-}$ 1) were used for electrospinning. Scanning electron microscopy (SEM) was employed to characterize the fibers. As shown in Figure S1, the diameter of nanofibers increased as PLGA concentration and the electrospinning speed increased. At a flow rate of $0.1 \mathrm{~mL} \mathrm{~h}^{-1}$, the average size of the fibers increased from $100 \mathrm{~nm}$ to $1040 \mathrm{~nm}$ as PLGA concentration changed from $3 \%$ to $15 \%$. When the flow rate increased to $0.3 \mathrm{~mL} \mathrm{~h}^{-1}$, obvious growth in the diameters of fibers was detected across the tested concentrations. The average diameters were ranged from $500 \mathrm{~nm}$ to $1930 \mathrm{~nm}$. Further increase in flow rate to $0.5 \mathrm{~mL} \mathrm{~h}^{-1}$ lead to the preparation of larger fibers. Under this electrospinning speed, fibers of average size ranging from $770 \mathrm{~nm}$ to $1950 \mathrm{~nm}$ can be obtained by varying PLGA concentration from $7 \%$ to $15 \%$. 

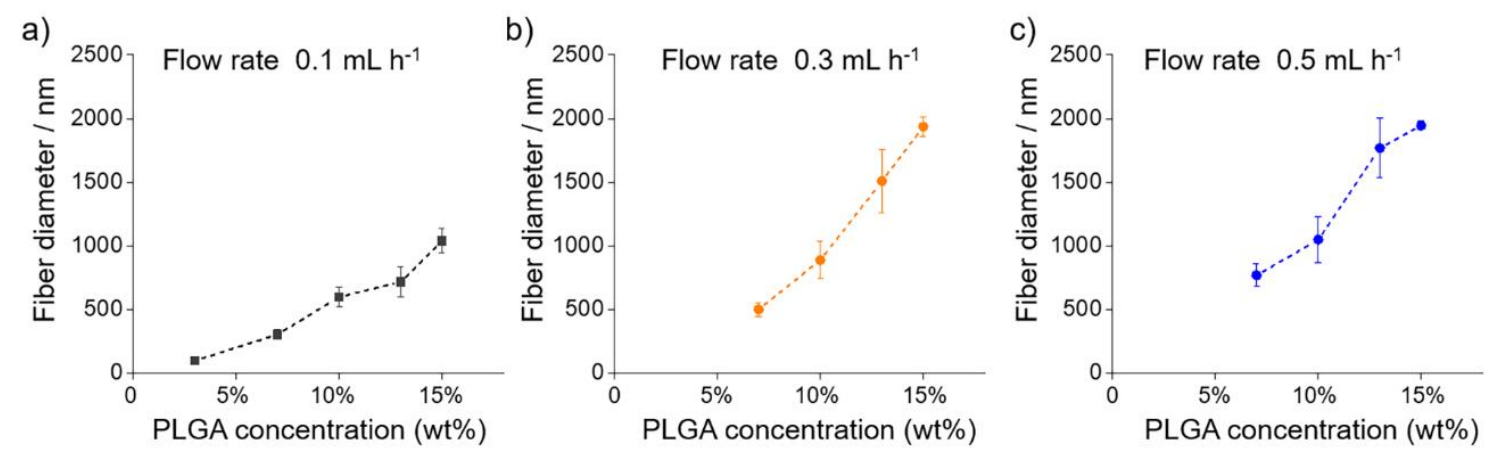

Figure S1. Effects from PLGA concentration and electrospinning rate on fiber diameters. Electrospinning flow rates of 0.1 (a), 0.3 (b), and 0.5 (c) $\mathrm{mL} \mathrm{h}^{-1}$ were examined, respectively.
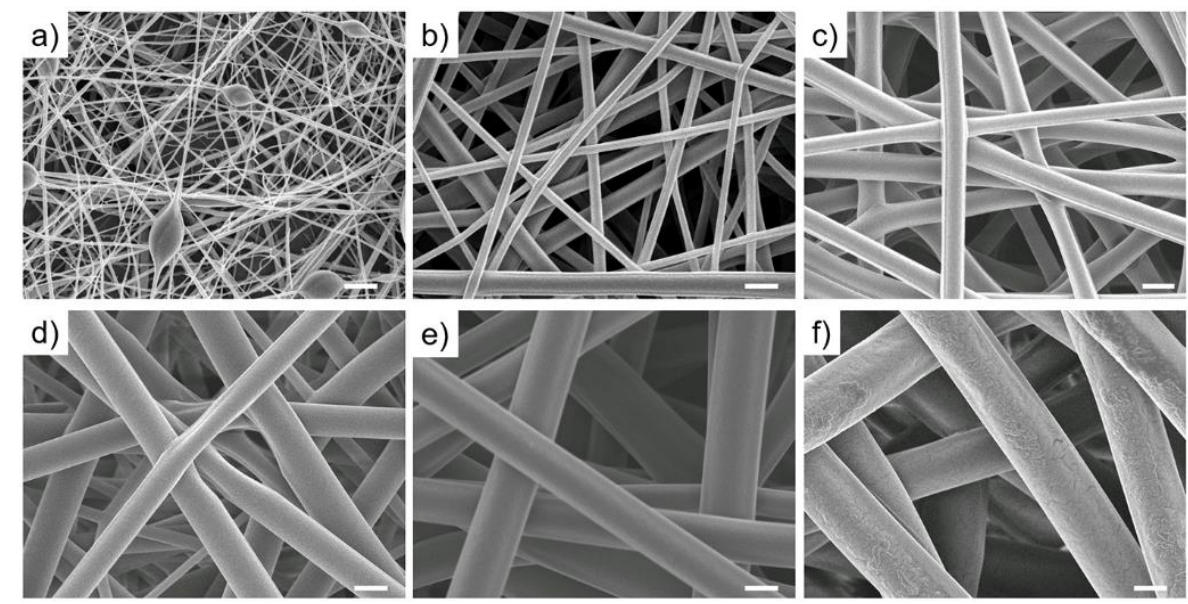

Figure S2. SEM characterization of PLGA fibers with varied diameters. The average diameters of the fibers are estimated as $100 \mathrm{~nm}$ (a), $300 \mathrm{~nm}$ (b), $770 \mathrm{~nm}$ (c), $1000 \mathrm{~nm}$ (d), $1500 \mathrm{~nm}$ (e) and $2000 \mathrm{~nm}$ (f). Scale bar: $1 \mu \mathrm{m}$.
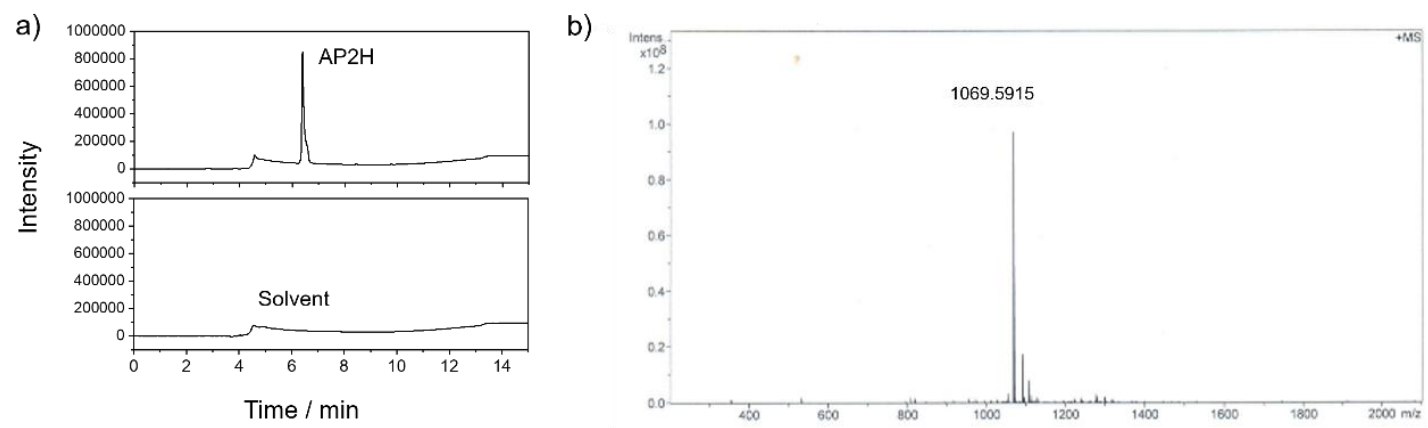

Figure S3. HPLC analysis and HR-MS characterization of purified AP2H. (a) HPLC chromatograms of AP2H and the solvent. Column: shim-pack XR-ODS column $(75 \times 4.6 \mathrm{~mm})$; wavelength: $220 \mathrm{~nm}$. Gradient: 0-10-15 min, 5\%B-90\%B-90\%B (A: $\mathrm{H}_{2} \mathrm{O}$ containing 0.1\% TFA, B: acetonitrile containing 0.1\% TFA). (b) HR-MS spectrum of AP2H (MALDI, positive mode): $\mathrm{m} / \mathrm{z}[\mathrm{M}+\mathrm{H}]^{+}$calcd: 1069.5907 , found: 1069.5915 . 


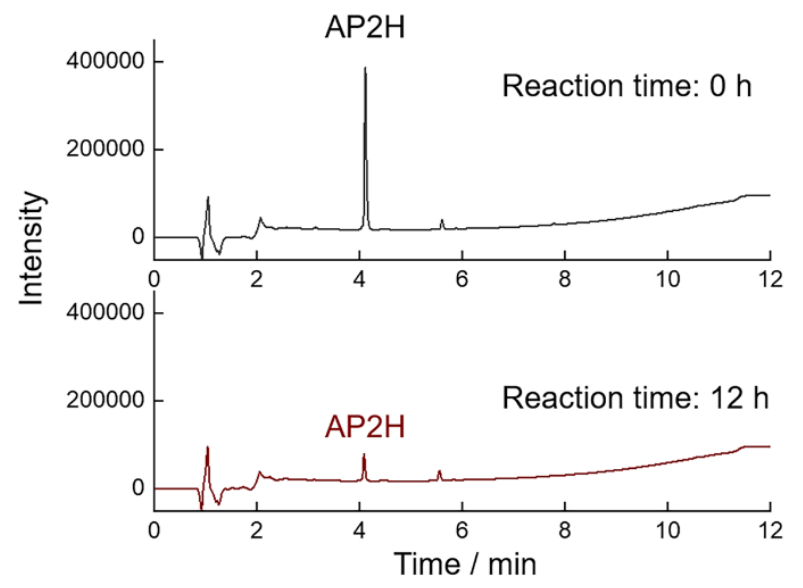

Figure S4. HPLC monitoring the change in AP2H content in the reaction solutions during the conjugation of AP2H. Column: shim-pack XR-ODS column $(75 \times 4.6 \mathrm{~mm})$; wavelength: 220 nm. Gradient: 0-10-15 min, 5\%B-90\%B-90\% B (A: $\mathrm{H}_{2} \mathrm{O}$ containing 0.1\% TFA, B: acetonitrile containing $0.1 \%$ TFA).

a)

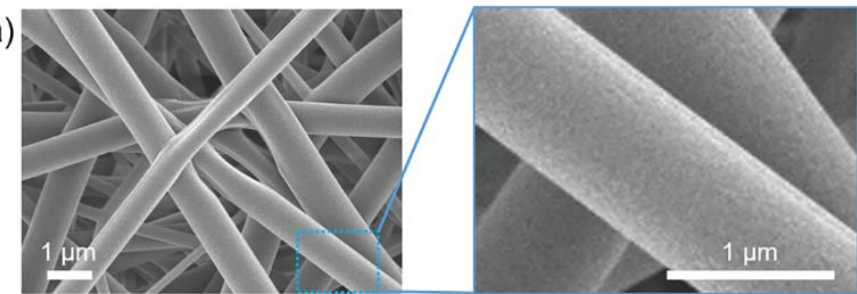

b)

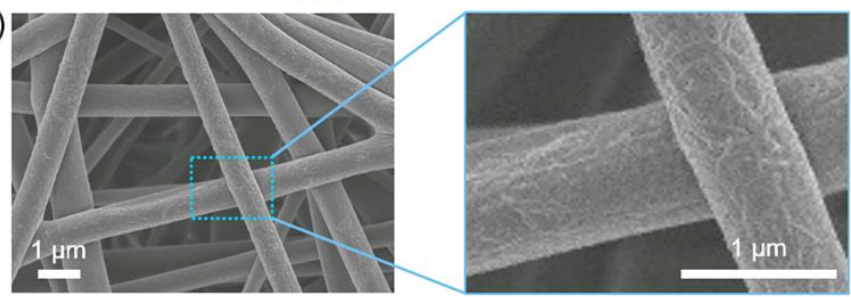

Figure S5. SEM characterization of PLGA nanofibers before (a) and after (b) peptide modification processes. 


\subsection{Fabrication of control substrates and their cell capture performance}

Control substrates with different surface modifications were prepared (Figure S6). Control substrate-1 was synthesized by immobilizing the disulfide bond-containing linker (SPDP) to PLGA fibers but without subsequent decoration of AP2H peptide. For control substrate-2, peptide AP2H was directly conjugated to the nanofibers via its amino terminal to the carboxyl group on PLGA fibers without any linker. The cell capture performance of these substrates was investigated by the incubation with HepG 2 cells. Without the functionalization of targeting peptide, control substrate-1 exhibited a capture efficiency of only 47\% (Figure S6). The low capture efficiency ruled out the affinity from the linker molecule to HepG2 cells. For control substrate-2, the cell capture efficiency was 62\% (Figure S6), suggesting the essential contribution of peptide AP2H to the binding of HepG2 cells. Compared with Si@PLGANF@AP2H, the compromised capture performance of control substrate-2 is most probably caused by the steric hindrance during the binding towards the cells. The direct attachment of peptide to the nanofibers inhibited the free movement and reduced the accessibility toward its target protein on cell membrane.

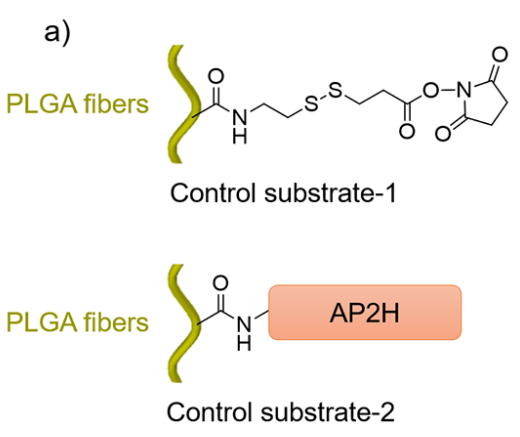

b)

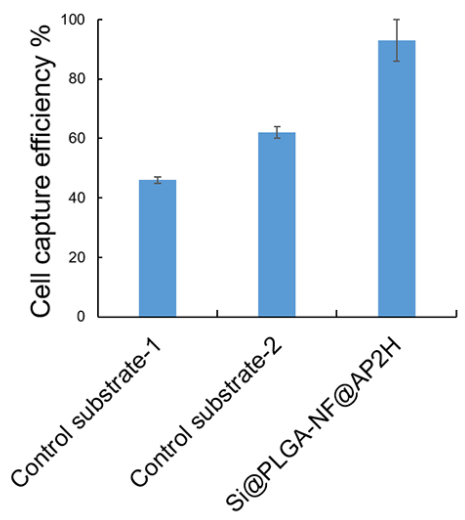

Figure S6. (a) Illustration of the compositions of control substrates. (b) Cell capture efficiencies of different substrates toward HepG2 cells. 


\subsection{Cell binding kinetics on Si@PLGA-NF@AP2H substrate}

Cancer cells from liver (HepG2) and breast (MCF-7) carcinomas, as well as glioblastoma (U251) were subjected to the incubation with peptide-functionalized nanointerfaces (Si@PLGA-NF@AP2H) for 15, 30, 45, and 60 min, respectively. As shown in Figure S7, the capture efficiencies increased as the incubation time increased. After 15-min incubation, the average capture efficiencies of all three kinds of cancer cells were above 60\%. These data reached $80 \%$ when the incubation time elongated to $30 \mathrm{~min}$. For MCF-7, the binding to Si@PLGA-NF@AP2H reached equilibrium after 45-min incubation with a capture efficiency of $96 \%$. Further increase of incubation time to $60 \mathrm{~min}$ did not change the result. In comparison, the capture efficiencies toward HepG2 and U251 cells were still around 80\% when the incubation time was $45 \mathrm{~min}$. HepG2 and U251 cells need $60 \mathrm{~min}$ to achieve high capture efficiencies (>90\%). These results suggest that different cancer cells may display varied binding kinetics to the substrate. For some cancer cells such as MCF-7, a shorter incubation time is enough to obtain high capture performance. In this work, to ensure the binding equilibrium and high capture efficiency toward different cancer cells, $60 \mathrm{~min}$ was chosen as the optimized incubation time.

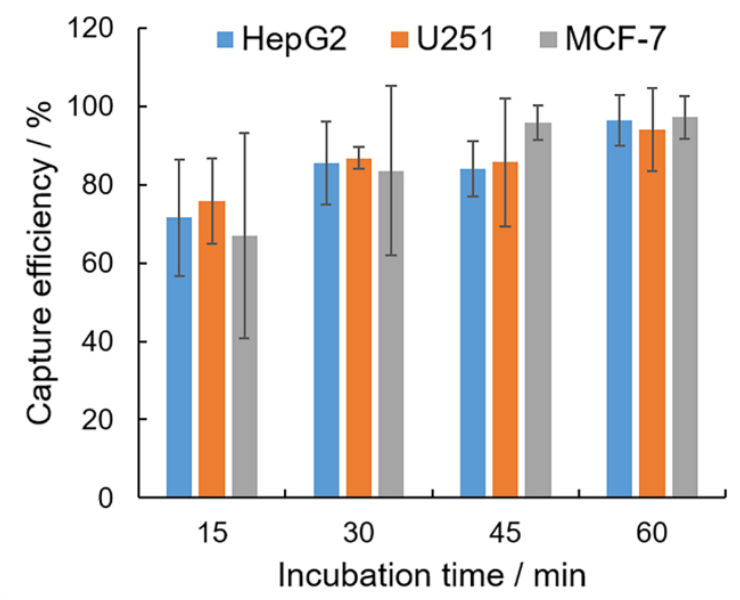

Figure S7. The effect of incubation time to capture efficiencies of Si@PLGA-NF@AP2H toward HepG2, U251 and MCF-7 cells. 
2.4 Characterization of LAPTM4B and EPCAM in HEK293 and HCC cell lines

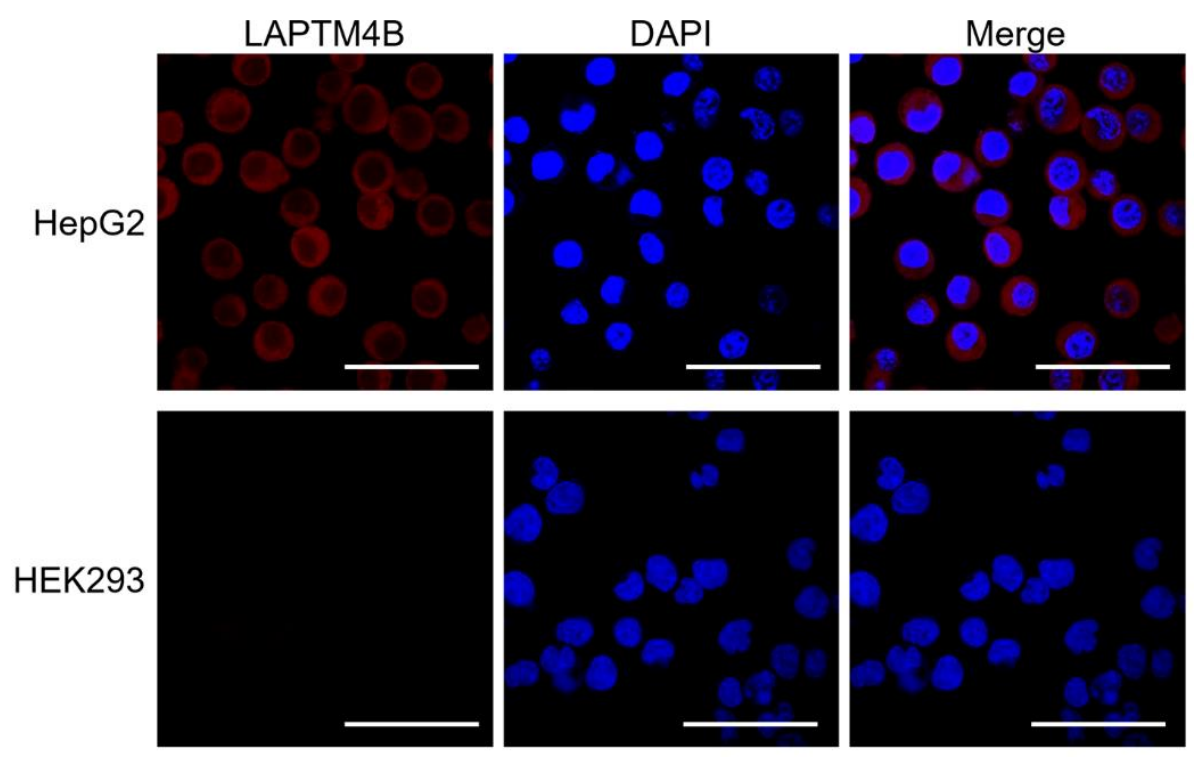

Figure S8. Immunofluorescence staining of LAPTM4B in cancer (HepG2) and normal (HEK293) cells. Scale bar: $50 \mu \mathrm{m}$.

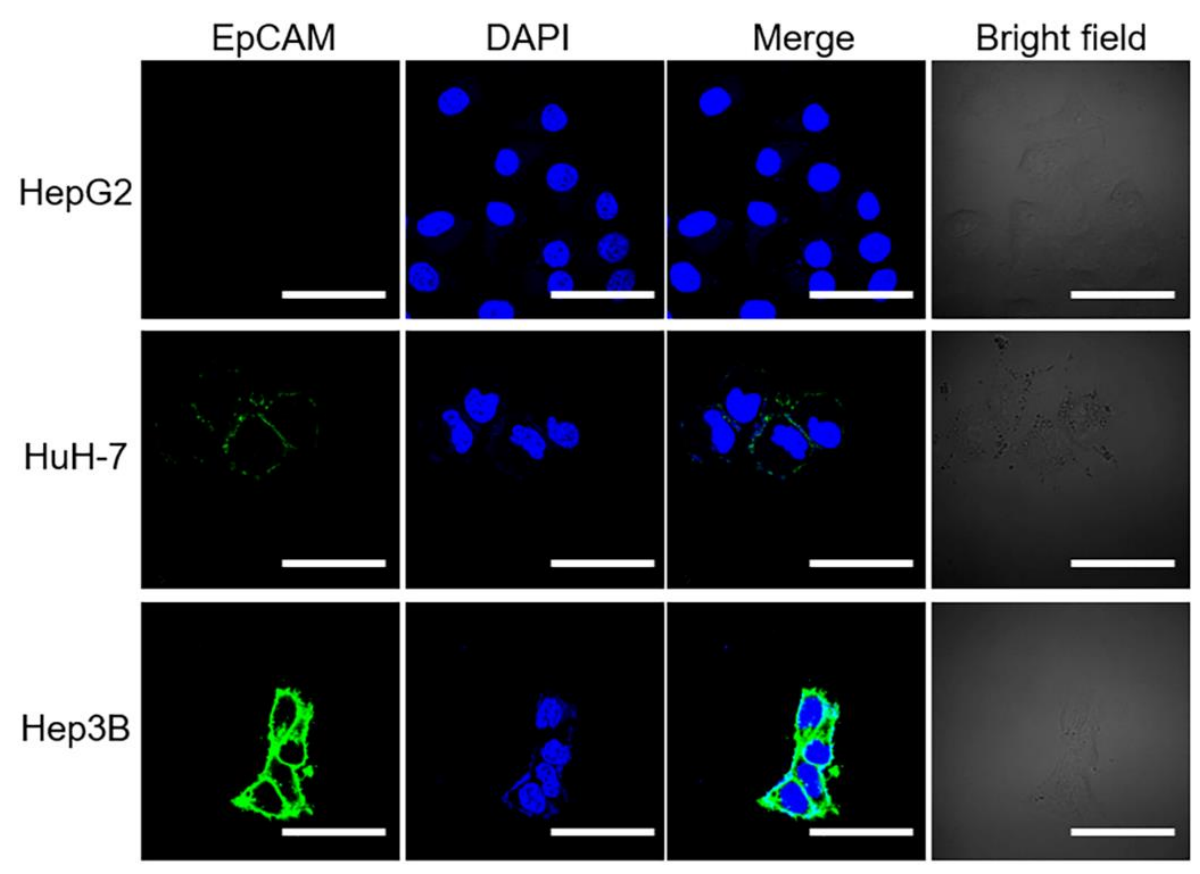

Figure S9. Immunofluorescence staining of EpCAM in HepG2, HuH-7 and Hep3B cells. Scale bar: $50 \mu \mathrm{m}$. 


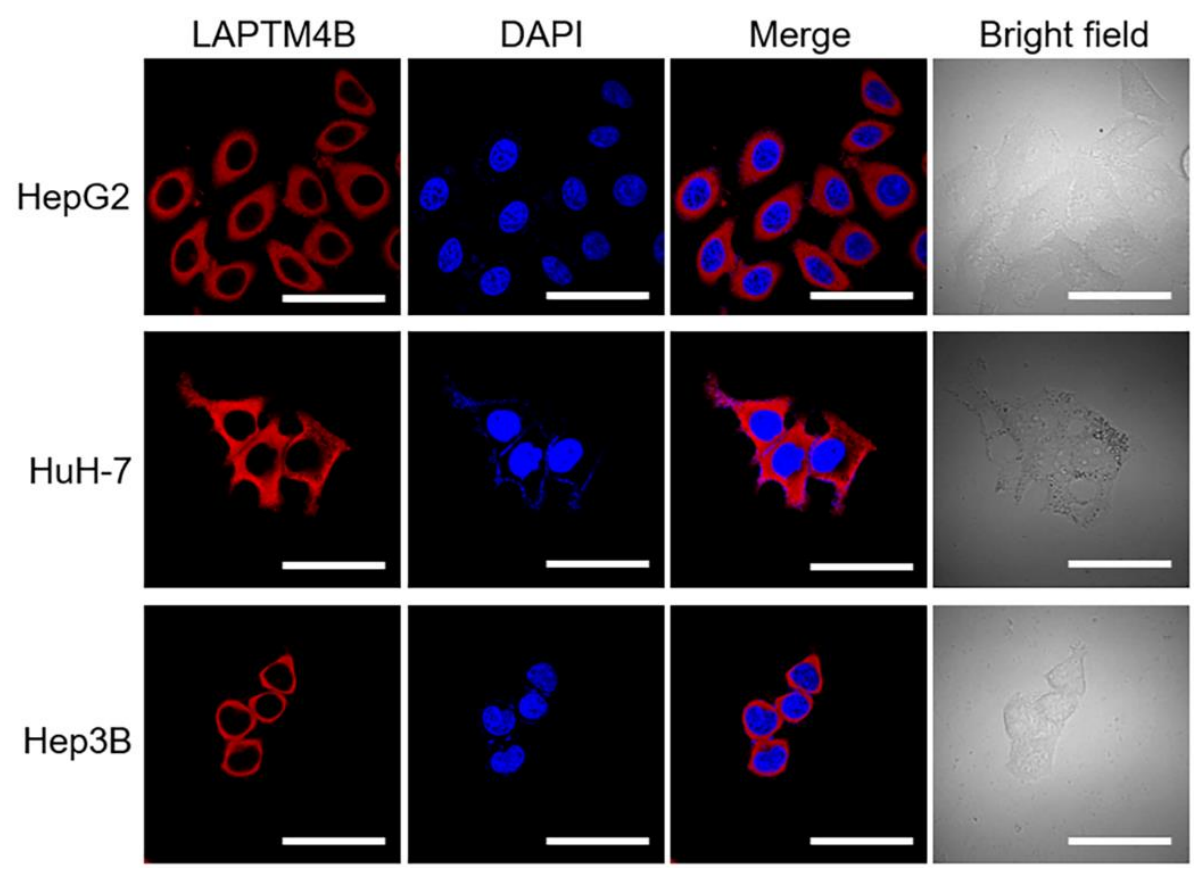

Figure S10. Immunofluorescence staining of LAPTM4B in HepG2, HuH-7 and Hep3B cells. Scale bar: $50 \mu \mathrm{m}$.

\subsection{Determination of peptide binding affinity toward HuH-7 and Hep3B cells}

a)

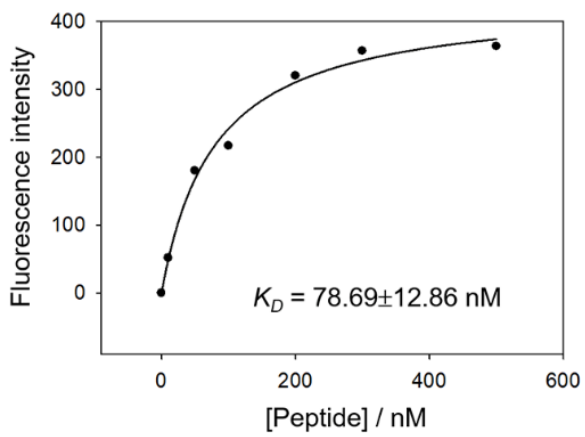

b)

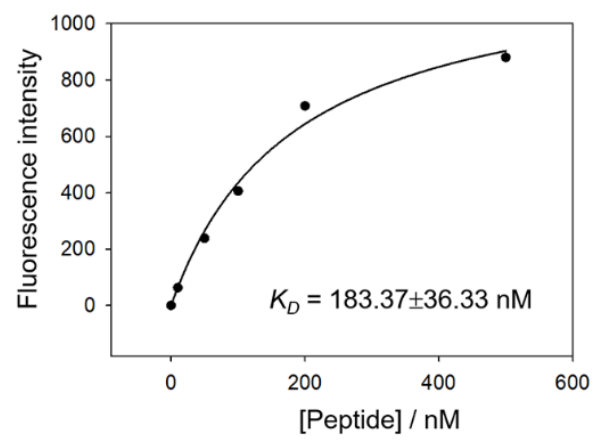

Figure S11. Fitting of binding affinity of AP2H toward HuH-7 (a) and Hep3B (b) cells. 


\subsection{Characterization of EpCAM in MCF-7 and U251 cells}

a)

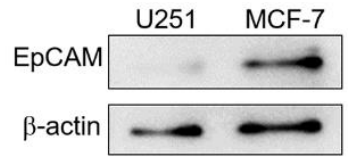

b)

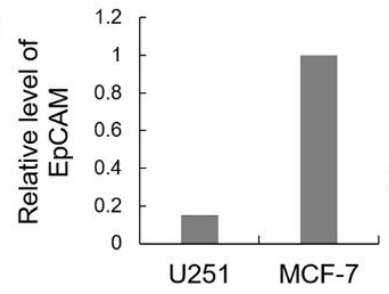

c)

EpCAM DAPI

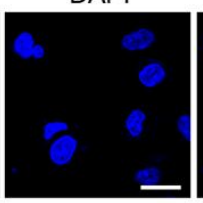

Merge

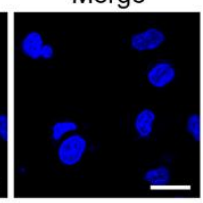

MCF-7

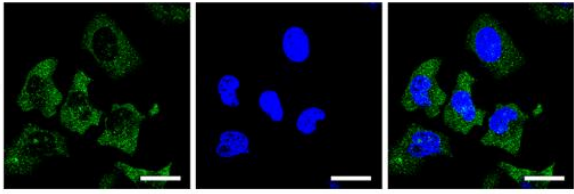

Figure S12. (a) Western blotting of EpCAM in U251 and MCF-7 cells. (b) Quantitative analysis of the expression of EpCAM based on western blotting results. $\beta$-actin was used as the internal reference. (c) Immunofluorescence staining of EpCAM in U251 and MCF-7 cells. Scale bar: $10 \mu \mathrm{m}$.

\subsection{Capture of $\mathrm{HCC}$ cells with different EpCAM levels from whole blood}

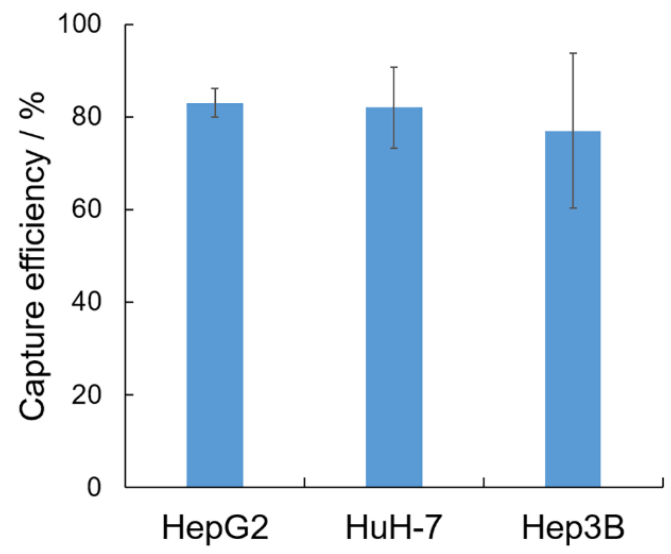

Figure S13. Capture performance of Si@PLGA-NF@AP2H toward HepG2, HuH-7 and Hep3B cells in artificial patient blood samples (spiked: 50 cells $\mathrm{mL}^{-1}$ ). 


\subsection{Ligand exchange and cell release efficiencies of GSH}

To examine the ligand exchange efficiency of GSH, a peptide conjugate (AP2H-SPDP) possessing the same molecular structure as the moiety decorated on PLGA fibers was firstly synthesized by a condensation reaction between AP2H peptide and the disulfide bondcontaining linker (SPDP) (Figure S14a). The reaction between GSH and AP2H-SPDP was monitored with HPLC. As shown in Figure S14c, after the treatment with GSH for only 1 min, a new peak identified as the product appeared (product 1, for its structure please see Figure S14b), suggesting the cleavage of SPDP from the peptide conjugate by GSH. As the reaction time prolonged to $5 \mathrm{~min}$, the signal of the product 1 increased, while the peak of AP2H-SPDP decreased. After $10 \mathrm{~min}$, the conversion ratio (95\%) from AP2H-SPDP to 1 kept almost the same as that obtained with a reaction time of $5 \mathrm{~min}(95 \%)$, suggesting the equilibrium of the reaction. Further prolongation of reaction time to $30 \mathrm{~min}$ can hardly increase the conversion efficiency (96\%). Based on these results, GSH can cleave $95 \%$ of the peptide conjugate AP2HSPDP in $5 \mathrm{~min}$, demonstrating the high reaction efficiency of GSH in solution.

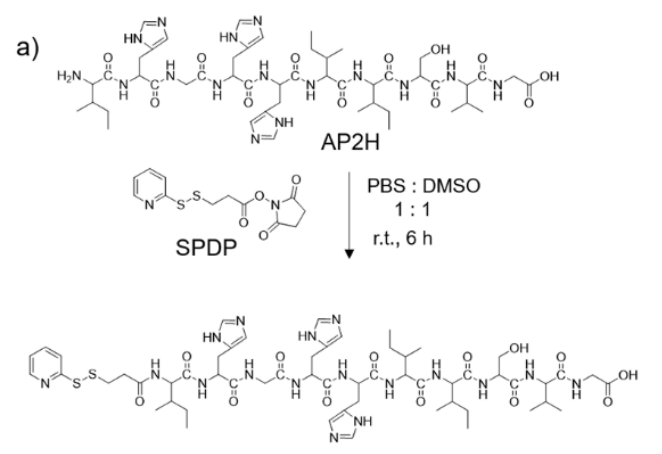

AP2H-SPDP

c)

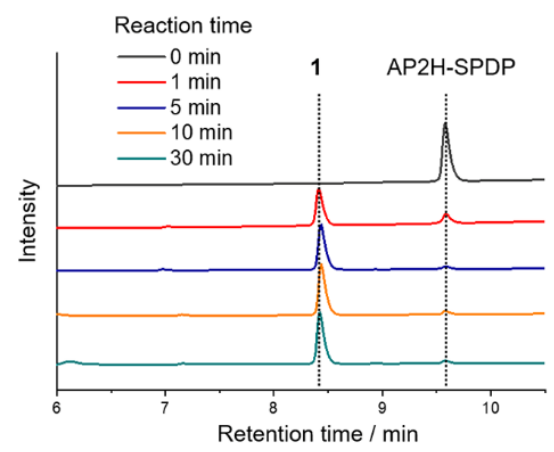

b)
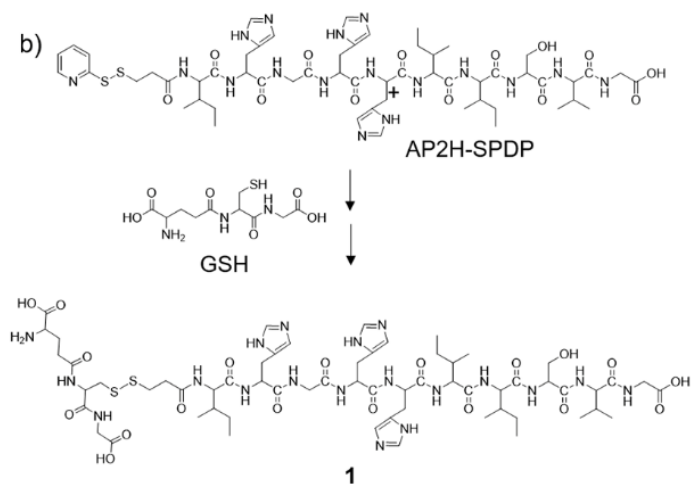

d)

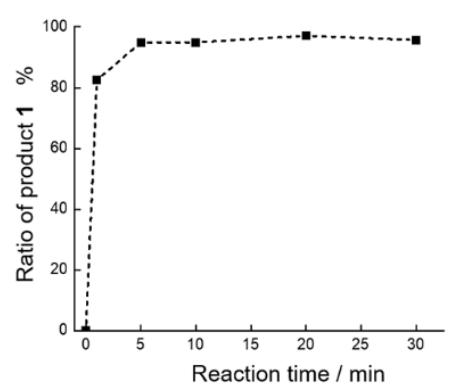

Figure S14. (a) Synthesis of peptide conjugate AP2H-SPDP which has the same molecular structure as the moiety decorated on PLGA fibers. (b) Reaction of AP2H-SPDP with GSH in solution affording a product 1. (c) Monitoring the reaction between AP2H-SPDP and GSH with HPLC. (d) Plotting of the ratio of product 1 versus the reaction time. 
The release efficiency of GSH toward the captured cells on the Si@PLGA-NF@AP2H substrate was also examined. Treatment with GSH released the captured HepG2 cells from the substrate via the exchange of the disulfide bond. The release ratios of the captured cells after treated with GSH for 10, 20, 30 and 40 min were calculated. As shown in Figure S15a, 10-min treatment with GSH resulted in the release of $47 \%$ captured HepG2 cells. As the treatment time increased, the release ratios increased as well. After treated with GSH for $30 \mathrm{~min}$, the average release ratio reached $82 \%$. Further increase of the incubation time to $40 \mathrm{~min}$ did not change the release ratio (Figure S15a). These results demonstrate the equilibrium of deoxidization reaction between GSH and the disulfide bonds on the substrate. Therefore, $30 \mathrm{~min}$ was chosen as the optimized reaction time to release the captured cells by GSH cleavage.

The GSH-based cell release was also compared with the conventional trypsin digestion approach. ${ }^{1}$ For HepG2 cells captured in PBS, the release efficiencies were $85 \%$ and $80 \%$ by using GSH and trypsin respectively (Figure S15b). To mimic the clinical samples, artificial blood samples were also examined. HepG2 cells captured in the whole blood were treated with GSH (1 mg mL $\left.\mathrm{m}^{-1}, 30 \mathrm{~min}\right)$ and trypsin $\left(2.5 \mathrm{mg} \mathrm{mL}^{-1}, 1 \mathrm{~min}\right)$, respectively. As shown in Figure S15b, GSH exhibited a cell release efficiency of $78 \%$. In comparison, a significant decrease in cell release efficiency was detected for trypsin. After trypsin digestion, only $50 \%$ of the captured HepG2 cells were released. This can be ascribed to the high complexity of the blood, and the low-specificity of trypsin digestion. Even little contamination from blood cells or serum proteins may act as competitive substrates for trypsin, thus reduced the digestion and release of the captured HepG2 cells. In this work, the introduction of a disulfide bond-containing spacer during peptide modification allows specific and mild release of intact cells from the substrate via ligand exchange.
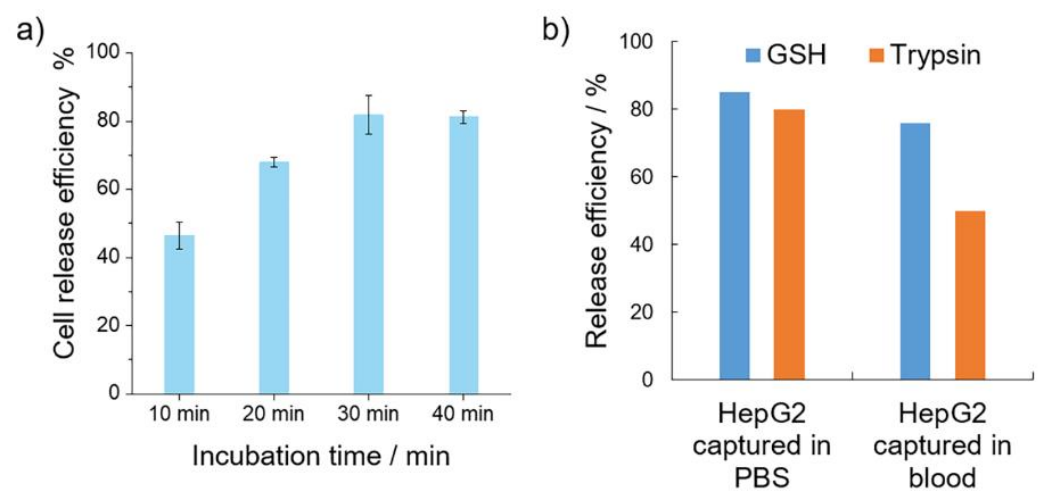

Figure S15. (a) Examination of cell release efficiency of GSH with varied incubation time. (b) Cell release efficiencies toward HepG2 cells captured in PBS and in blood respectively by using GSH and trypsin. 


\subsection{In vitro culture and proliferation activity of released cells}

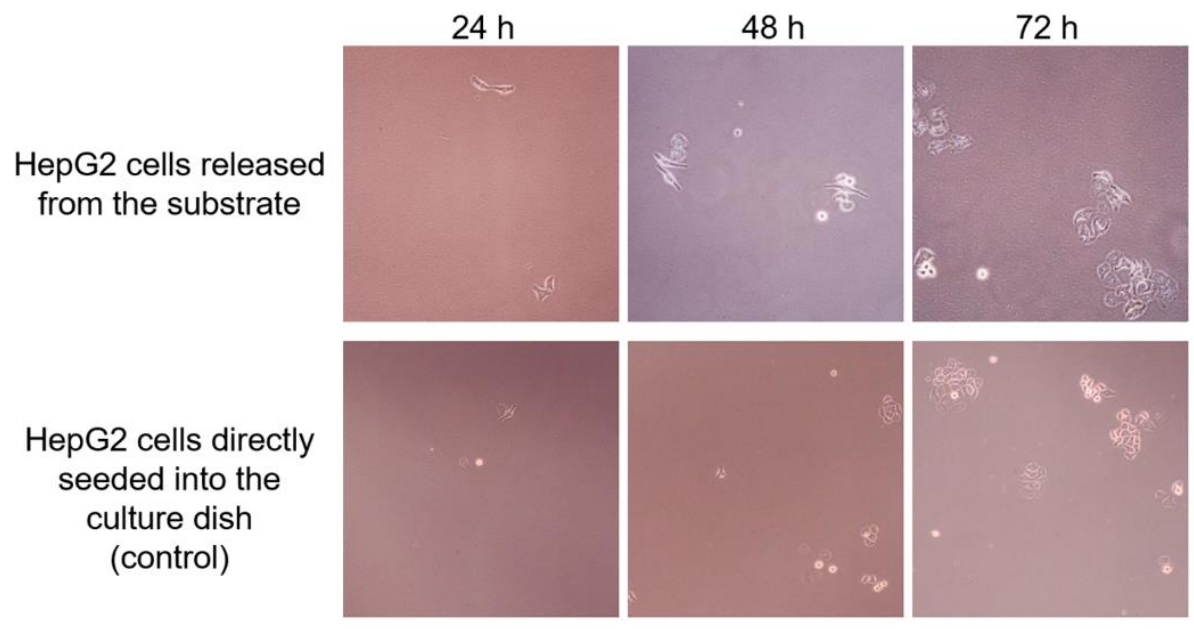

Figure S16. Observation of adhesion and proliferation activity of HepG2 cells released by GSH from Si@PLGA-NF@AP2H substrate. HepG2 cells directly seeded into the culture dish with the same density were used as a control.

\subsection{CTC and CTC clusters captured from the blood samples of HCC patients}

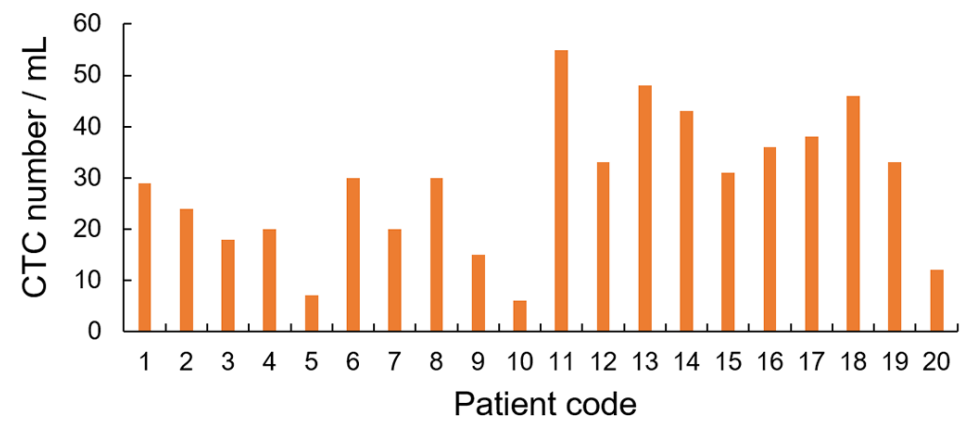

Figure S17. Quantification of CTCs in the blood samples from $20 \mathrm{HCC}$ patients. 


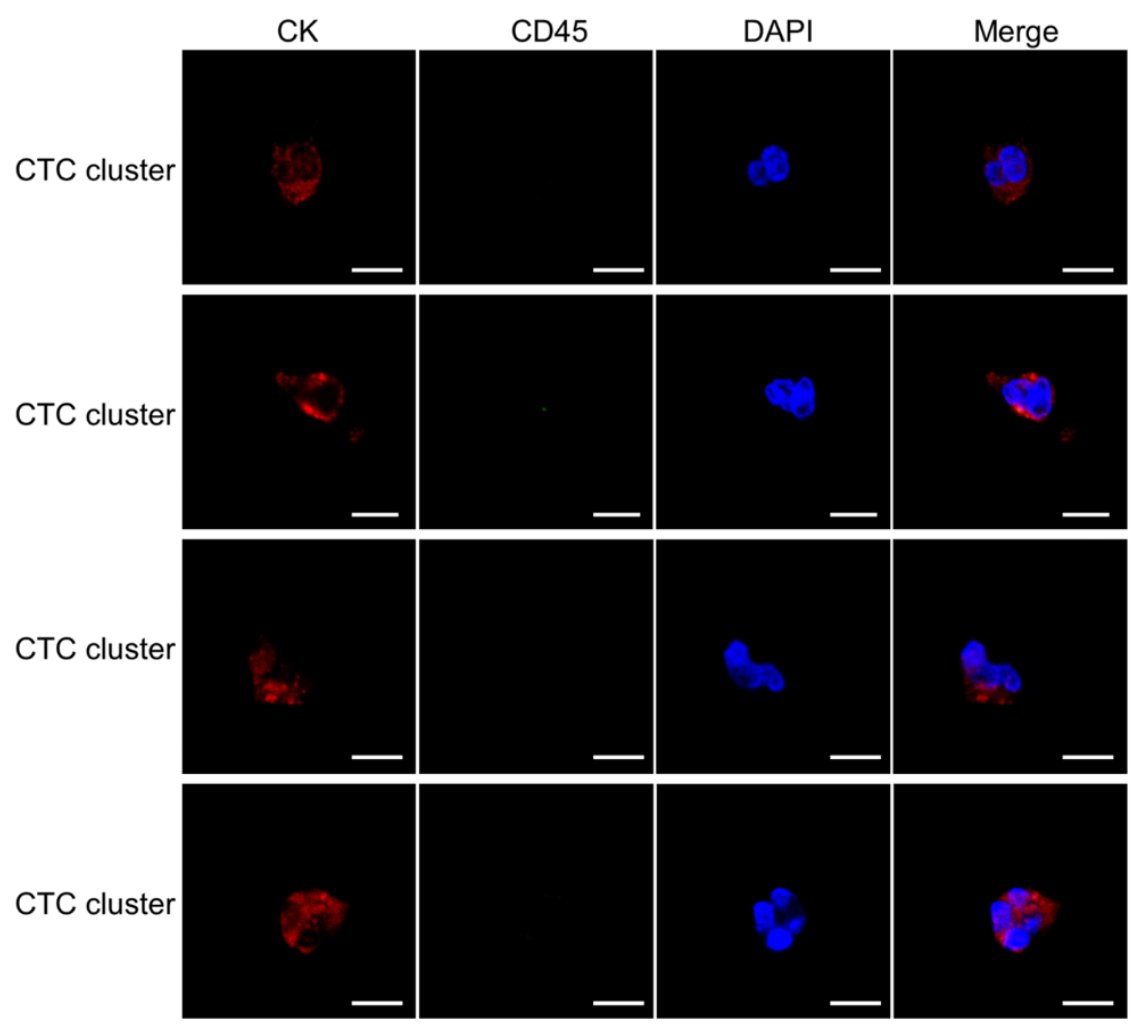

Figure S18. CLSM images of CTC clusters captured from the blood samples from HCC patients. Three-color immunocytochemistry method based on a nuclear indicator DAPI, eFluor $^{\mathrm{TM}} 570-$ labeled anti-CK and FITC-labeled anti-CD45 was used to identify CTCs and WBCs on Si@PLGA-NF@ AP2H. Scale bar: $10 \mu \mathrm{m}$.

\subsection{Analysis of CTCs captured from patient blood samples based on EpCAM level}

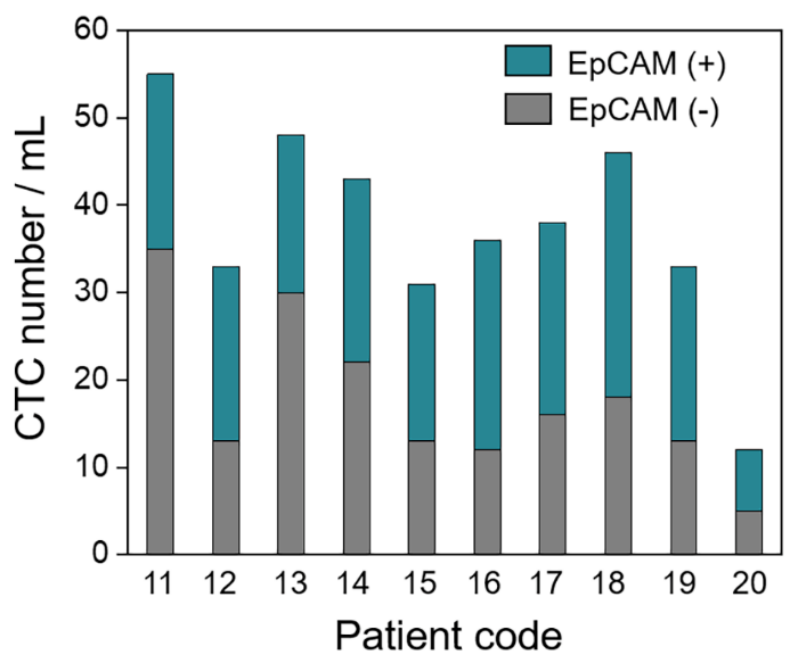

Figure S19. Numbers of CTCs with high and low EpCAM expression levels captured from HCC patients. 


\subsection{LAPTM4B expression in CTCs captured from patient blood samples}

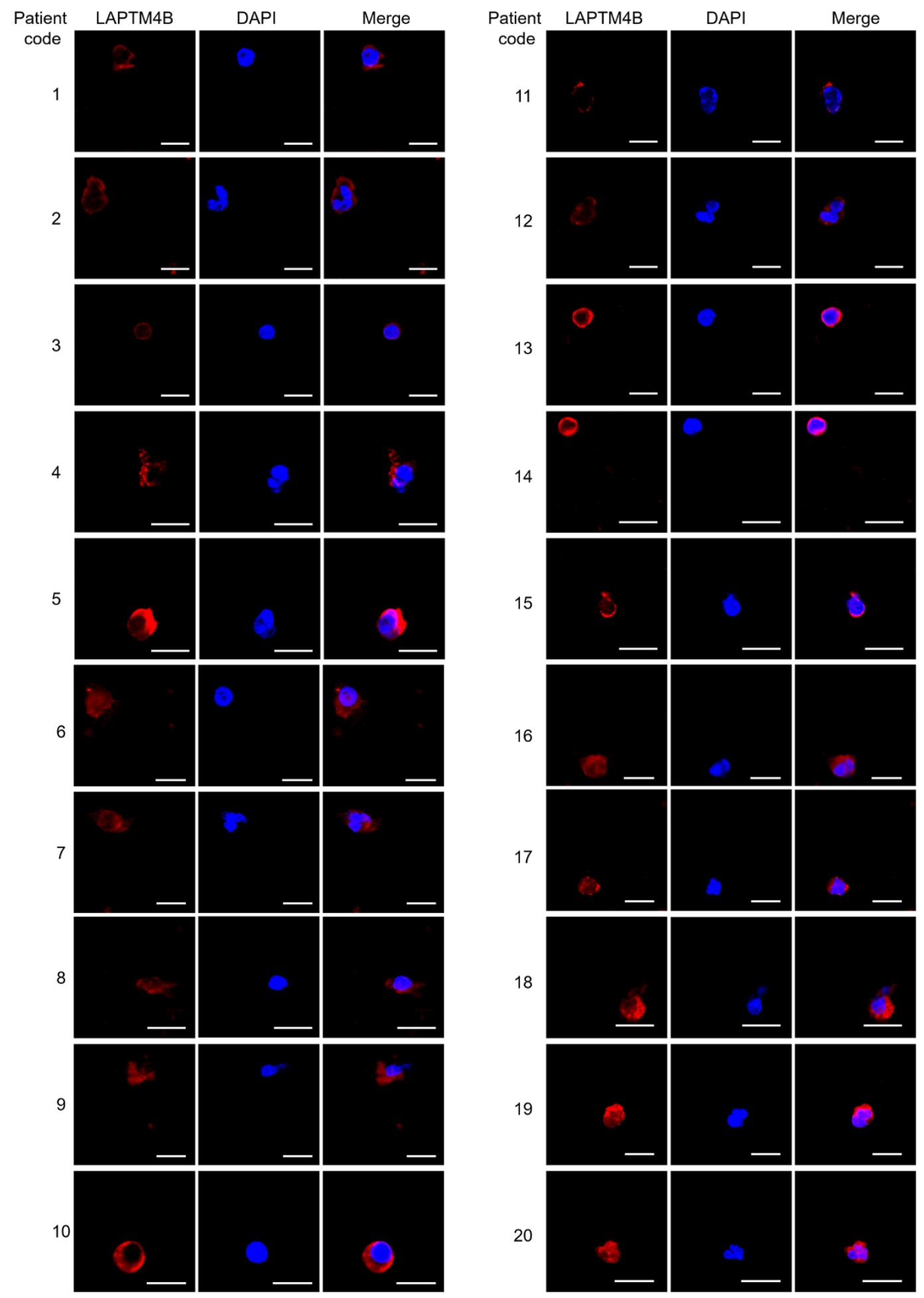

Figure S20. Immunofluorescence staining of LAPTM4B in the captured CTCs from the blood samples of $20 \mathrm{HCC}$ patients. Scale bar: $10 \mu \mathrm{m}$. 


\subsection{3}

Release of CTCs captured from patient blood samples

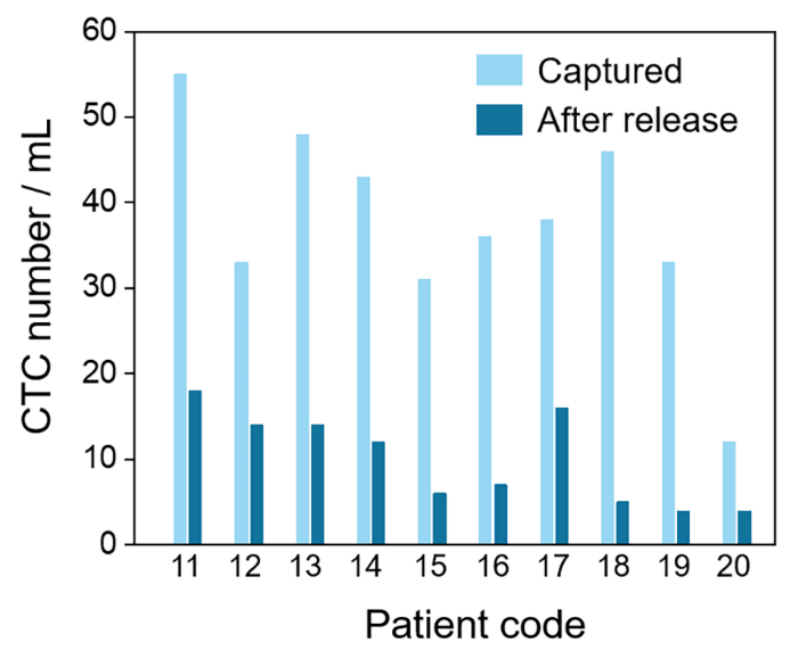

Figure S21. Release performance of captured CTCs from patient blood samples.

\subsection{Characterization of LAPTM4B expression in CTCs captured from patients} during therapy
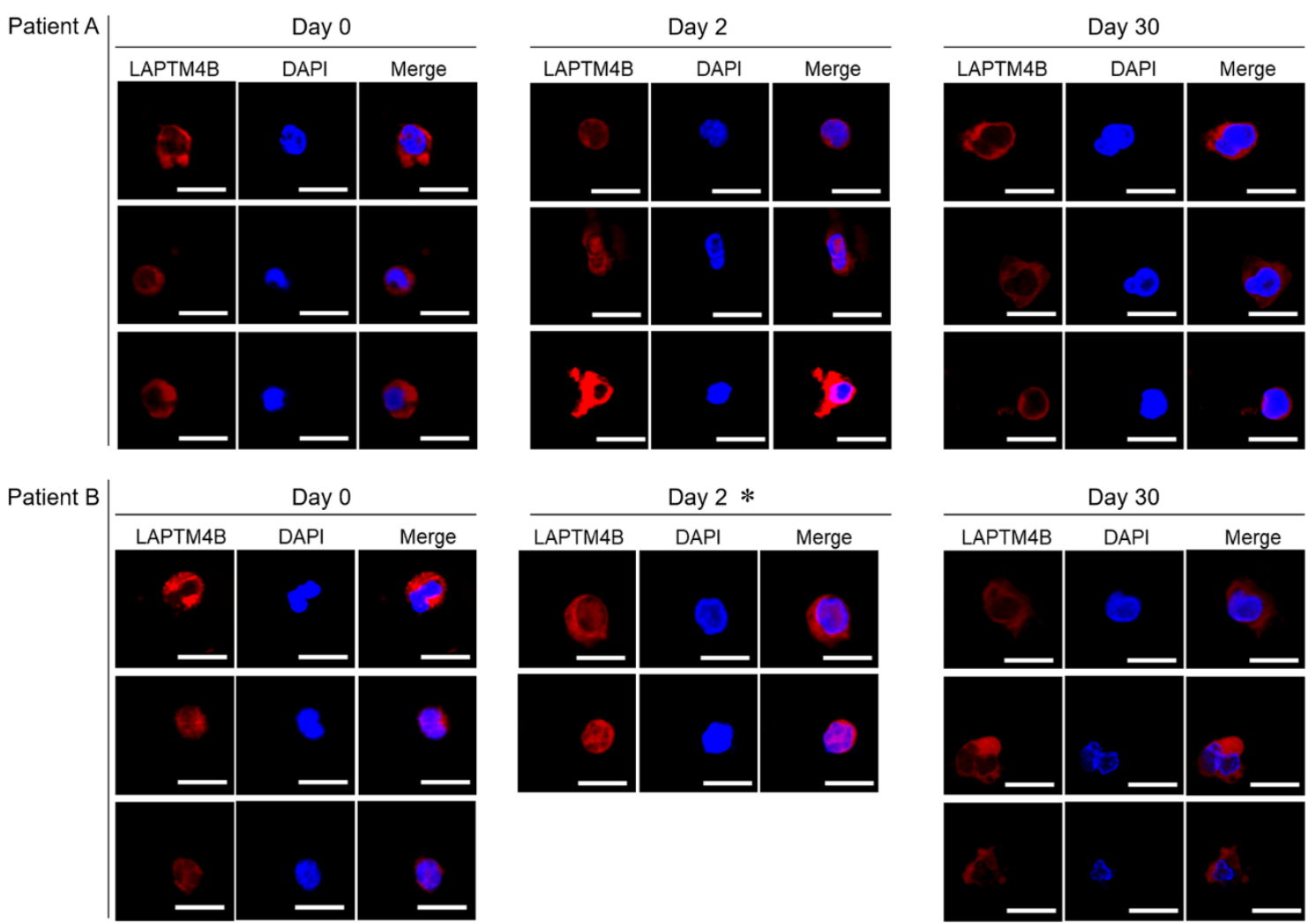

Figure S22. Representative immunofluorescence images of the expression of target LAPTM4B protein in the captured CTCs from the blood samples of Patients A and B during treatment. Scale bar: $10 \mu \mathrm{m} .{ }^{*}$ For Patient B, only 2 CTCs were detected in $1 \mathrm{~mL}$ blood sample on the second day after treatment. 


\section{References}

(1) Luan, C.; Wang, H.; Han, Q.; Ma, X.; Zhang, D.; Xu, Y.; Chen, B.; Li, M.; Zhao, Y. ACS Appl. Mater. Interfaces 2018, 10, 21206-21212. 